\title{
Vision-based detection of container lock holes using a modified local sliding window method
}

\author{
Yunfeng Diao ${ }^{1,2}$, Wenming Cheng ${ }^{1,2}$, Run $\mathrm{Du}^{1,2^{*}}$ (D), Yaqing Wang ${ }^{3}$ and Jun Zhang ${ }^{1,2}$
}

\begin{abstract}
Container yards have been facing the increase of freight volume. In order to improve the efficiency of container handling, automatic stations have been established in many terminals. However, current container handling still needs a manual operation to locate container lock holes. Hence, it is inefficient and potential to risk workers' health under long working hours. This paper presented a hybrid machine vision method to automatically recognize and locate container lock holes. The proposed method extracted the top area of the container from the multiple container areas, and then presented a new modified local sliding window to detect the keyhole region. The algorithm learned the histograms of oriented gradients (HOG) features using a multi-class support vector machine (SVM). Finally, the holes were located using direct least square fitting of ellipses. We carried an experiment under various weather and light conditions including nights and rainy days. The results showed that both the recognition and location accuracy outperformed the state-of-the-art results.
\end{abstract}

Keywords: Container port, Computer vision, Container lock holes location, Automatic handling

\section{Introduction}

In the container terminal, the productivity of container handling immensely impacts the whole efficiency of the terminal because container handling operates more frequently than other logistics activities. During handling, crane operators need to observe the lock holes and align the spreader with the container with a long working time. Such manual operations are time-consuming and require high technique of operators. Besides, the cab is mounted below the crane girder, thus the driver has to keep his head down to observe the position of the container for a long time, which would risk operators' safety and health. With the fast growth of container trade volumes [1], there is a need for an efficient, safe, and, automated method to handle containers [2].

Over the past decade, many researchers have devoted themselves to improve the productivity of container handling by using laser scanning [3], anti-sway control

\footnotetext{
* Correspondence: rdu@swjtu.edu.cn

${ }^{1}$ School of Mechanical Engineering, Southwest Jiaotong University, Chengdu, China

${ }^{2}$ Technology and Equipment of Rail Transit Operation and Maintenance Key Laboratory of Sichuan Province, Chengdu, China

Full list of author information is available at the end of the article
}

[4, 5], and made remarkable achievements. However, these assistant methods mainly solve the problem of the location of the container truck and swing of the spreader respectively, which are difficult to apply to the container lock hole location. In recent years, with the prevalence of cameras in container terminals and the increasing development in the field of computer vision and machine learning, the automatic container location can be achieved based on vision methods. Li and Lee [6] proposed a method for recognizing the container shape and range, which detects the container contour by Hough transform and uses the stereo camera to measure the container depth information. However, detecting straight lines by Hough transform is easily interfered by surface texture and is not robust enough. Chen and Wang [7] proposed a container recognition and location method based on feature matching, which improves the effectiveness of detecting container's shape. Yoon and Hwang [8] got information about container position based on stereo vision. However, these methods focus on the whole container, which have problems in recognizing and locating the small and key targets, such as the corner casting and the hole. Shen and Mi [9] extracted the 
container lock holes by using traditional image processing methods. Mi and Zhang [10] recognized the right corners by using HOG, then the left corners could be obtained by symmetry.

Although some achievements have been done, some challenges still remain unsolved. (1) Most algorithms are based on the traditional image processing methods, which are not robust to container conditions, various weathers and illuminations, especially for the rusty keyholes, nights, and rainy days. (2) Those methods depend on high requirements for the shape of the container in the captured images, which contain only top area or even local regions. Thus, it is difficult to detect the lock holes from container images containing multiple surfaces.

Therefore, we proposed a combination of various machine vision algorithms to solve these challenges. In order to evaluate our method, the testing dataset contained container miniatures of rusty lock holes in various environments. Experimental results showed that the average accuracies of recognition were all $100 \%$ in any conditions. The average accuracies of location were $98.13 \%$ at daytime and $90.50 \%$ at night respectively, and all outperforms the state-of-the-art results. Additionally, the first proposed rainy experiment which achieved average accuracies of $100 \%$ and $95.25 \%$ for recognition and location respectively showed that the method was also adaptive to rainy days.

The rest of the paper is organized as follows. Section 2 describes the method framework, including the container extraction, hole recognition, and hole location. Section 3 gives out the dataset and experimental results and compares them with state-of-art results, then analyzes the causes of error. Section 4 concludes the paper.

\section{Methodology}

The overall framework of the proposed method is shown in Fig. 1. Firstly, the image is segmented by HSV (hue, saturation, value) color space and k-means clustering algorithm, then the container region is clipped from the whole image after affine transformation. Secondly, the fixed-size section of the container region which is clipped with a sliding window is detected by HOG features. Thirdly, a one-against-all SVM is adopted for classification. If the classification is successful, hole center locating starts; otherwise, it continues to the second step. The progress is shown in Fig. 2.

\subsection{Image segmentation based on HSV space \\ 2.1.1 Container segmentation}

The background environment is usually monotonous and unchanged during container handling, and the color of most containers is single and solid. Thus, the container can be segmented from the background by the color difference between the container and background. The RGB color model has many limitations when used for color description, for example, the three channels are highly correlated, and the difference between the two colors cannot be represented by the distance between two points in the color space. In contrast, the HSV color model is less sensitive to the external environment such as light, brightness, and shadow, and is easier to separate the object from the background. Thus, the image is transformed from RGB to HSV, obtaining the images of three channels of $\mathrm{H}, \mathrm{S}$, and $\mathrm{V}$ respectively.

$$
\begin{aligned}
& H=\left\{\begin{array}{c}
60(G-B) /(V-\min (R, G, B)), V=R \\
120+60(B-R) /(V-\min (R, G, B)), V=G \\
240+60(R-G) /(V-\min (R, G, B)), V=B \\
H+360, H<0
\end{array}\right. \\
& S=\left\{\begin{array}{c}
(V-\min (R, G, B)) / V, V \neq 0 \\
0, V=0
\end{array}\right. \\
& V=\max (R, G, B)
\end{aligned}
$$

To facilitate subsequent processing, the $\mathrm{R}, \mathrm{G}$, and $\mathrm{B}$ channels are normalized to the range $[0,1]$; hence, the $H$, $\mathrm{S}$, and V channels are within the range $[0,1]$. According to the experiment results, although the segmented container is completed in $H$ channel image, there are some noises in the background. In $S$ channel image, there are few noises, but the separation degree is lower between the object and the background, especially in sunny time or nighttime. Therefore, we combined $H$ and $S$ to a single channel for images captured in daytime, named $W$ :

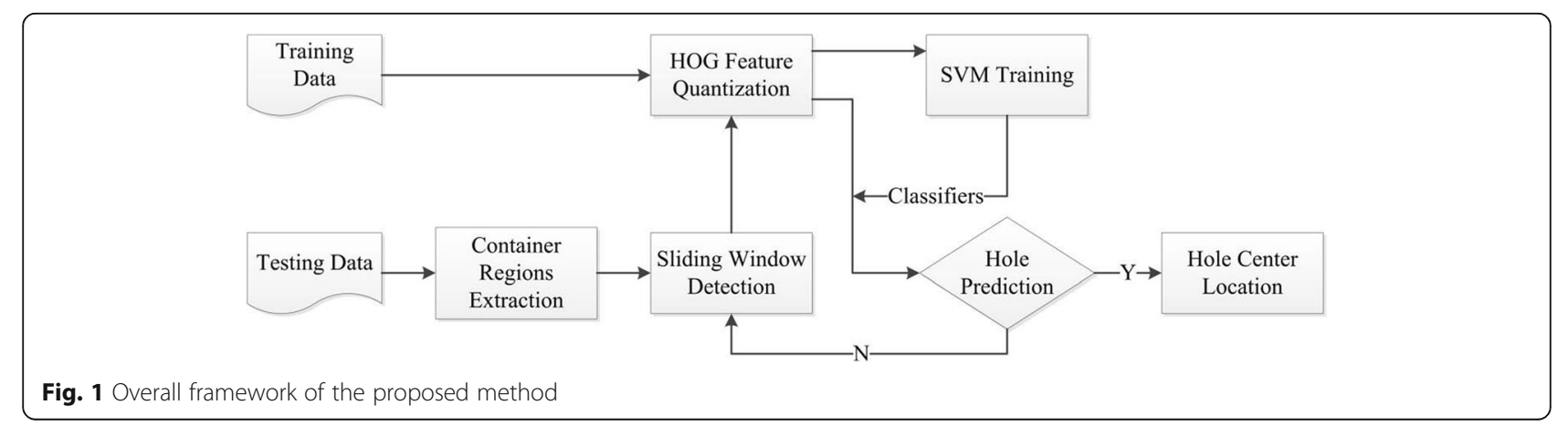




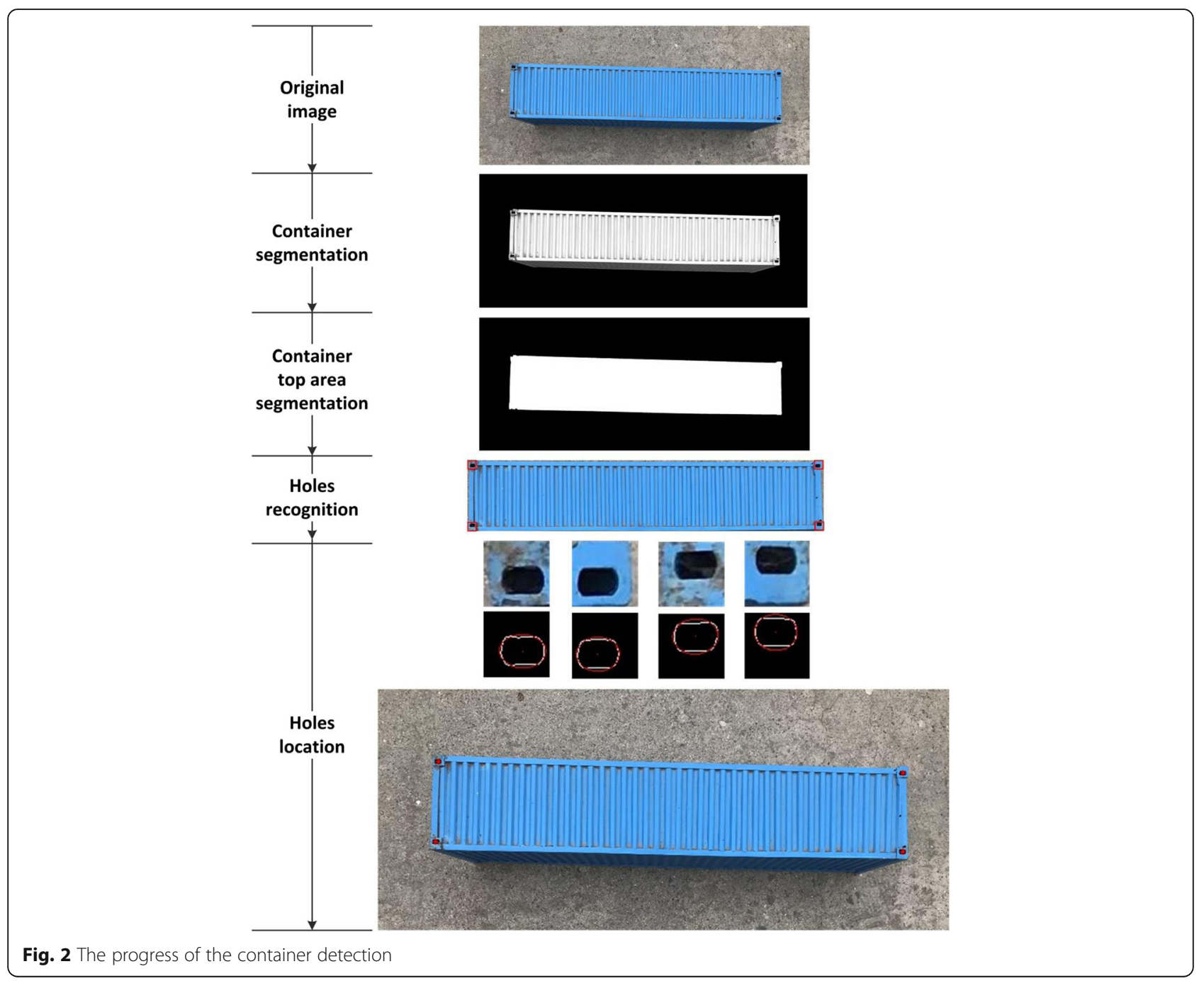

Fig. 2 The progress of the container detection

$$
W=0.3 H+0.7 S
$$

For images captured at night, the value of $W$ is equal to the $S$ channel. Because the obtained $W$ channel image still contains a little noise, we used median filtering for smoothing image. Then, we segmented the container and the background by binarization, and the global optimal threshold $T$ was selected by Otsu method [11]. The segmented image value $g(x, y)$ was calculated in Formula 3 , where $f(x, y)$ represented the value of $W$ channel.

$$
g(x, y)=\left\{\begin{array}{c}
1, f(x, y)>T \\
0 f(x, y) \leq T
\end{array}\right.
$$

After binarization, we used morphological processing [12] to eliminate the interfering points in the background and small holes in the object and to fill gaps in the contour.

\subsubsection{Container top area segmentation}

When taking pictures, the shapes of the container depend on the position and viewing angle of the camera. According to the actual shooting situation, there are two types of container in an image. One includes the top and side surfaces, and the other only has the top surface.

The top surface needs to be extracted from the container for images containing two surfaces since the lock holes in the top surface are identified for locating. For images taken at daytime, the k-means algorithm [13] is used to separate container top area by clustering the brightness value, because the brightness is largely different between the light-receiving surfaces and the lesslight-receiving surfaces. The clustering metric used Euclidean distance. Firstly, the value of $g(x, y)$ was replaced with Formula (4).

$$
g_{2}(x, y)=\left\{\begin{array}{c}
f_{v}(x, y), g(x, y)=1 \\
0, g(x, y)=0
\end{array}\right.
$$


Where $g_{2}(x, y)$ represented the replaced image, and $f_{v}(x, y)$ represented the channel $\mathrm{V}$ in the original image. According to the observation of the image of $g_{2}(x, y)$, it can be seen that the value was clearly divided into three clusters, so the cluster $\mathrm{K}$ was set to 3 . According to our experiment, the values of the initial cluster centroid are set to $0,0.5$, and 0.9. Finally, after the image was denoised by morphology, the cluster with the largest centroid was the top surface.

For the case that the container contains the only-top surface, the complete container top shape can still be obtained after adopting the above method, and it can eliminate the interfering points that cannot be eliminated in Section 3.1.1. For images taken at night, only the top surface illuminated by light has a high brightness. Therefore, the Ostu method can replace the k-means algorithm to binarize the $g_{2}(x, y)$ to obtain a complete top surface.

In order to extract the contour of the container top area, the image after segmentation was processed by Canny edge detector [14].

\subsubsection{Angle detection and position correction}

The location of the camera installation and container placement errors may change the angle of the container in the image. In order to correct the inclined errors of the container, it is necessary to perform angle detection and position correction on the container. The correction was obtained by solving the minimum area bounding rectangle of an enclosing contour. The algorithm principle and calculation steps were as follows:

(1) The convex hull of the contour was found. The number of the points on the convex hull was usually between 10 and 14 .

(2) The endpoints of the convex hull in the $x$ direction were denoted as $X \min$ and $X \max$, and the endpoints in the y directions were denoted as $Y \min$ and $Y \max$. The four points were connected to form an initial rectangular area, as the current minimum value.

(3) The rectangle rotates clockwise until one of its sides (side A) coincides with one edge of the convex hull. Then, the convex point of the farthest distance from the side and the leftmost and rightmost points in the direction of the side projection were found. Finally, a new rotated rectangle was generated, which enveloped these three points and one of its sides coinciding with side A.

(4) Calculate the new rectangular area and compare it with the current minimum value. If it was less than the current minimum value, the new rectangle was recorded as the smallest rectangle. If it was greater than or equal to the current minimum value, the original minimum rectangle was retained.

(5) Repeat steps (3), (4) until the angle of rotation was greater than $90^{\circ}$.
(6) Output the center position of the minimum rectangle as $\left(x_{c}, y_{c}\right)$ and the rotation angle $\theta$.

After rotation transformation, the minimum rectangle region, which was the top area, was extracted from the whole image.

\subsection{The recognition of the lock holes \\ 2.2.1 Detection of container features}

The key to the identification of locking holes is to detect the edge information of the hole contour. HOG feature extraction [15] can clearly describe the shape features around the lock holes and is robust to illumination information. Then, the HOG can be used in detecting the lock holes.

\subsubsection{Learning features and classification}

To learn and predict container features, a multiclass one-against-one SVM classifier was used [16]. The SVM is based on error-correcting output codes (ECOC) multiclass model [17]. It is more accurate than one-against-all SVM and faster than the ordinary one-against-one SVM.

When setting up the data set, we chose to clip a $50 \times 50$ pixel slices from the $\mathrm{M} 2 \times 425$ pixel container top images as the training images. Where M2 is the width, 425 is the height, and the size of M2 may vary depending on the size of the container and the effect of the segmentation. The lock holes can just fill the entire slice under such size. The data was divided into eight types, including "hole," "background," "half hole in vertical," "incomplete hole at left edge," "incomplete hole at right edge," "incomplete hole at upper edge," "incomplete hole at lower edge," "container." Figure 3 shows examples of the data types.

\subsubsection{Local sliding window recognition of lock holes}

After obtaining the trained model, a sliding window method was proposed to recognize the lock holes. In this paper, the traditional window sliding technology was improved so that the window only slid in the four corner areas of the image; thus, the times of window sliding was reduced. To ensure that the proportion of the lock holes in the sliding window was consistent with the data set, we scaled the container top image to M2 $\times 425$ pixels by following Formula (5):

$$
\left\{\begin{array}{c}
l=N / 425 \\
\mathrm{M} 2=\mathrm{M} \times \mathrm{L}
\end{array}\right.
$$

where $L$ is a scaling factor, and $M$ and $N$ are the width and height of the image. The four corner areas of the image were named as the block to slide the window within the block (Fig. 4). The block size was set to $W_{1} \times W_{2}$ pixels, ensuring that the four lock holes of the container were all within blocks. The method was as follows: 

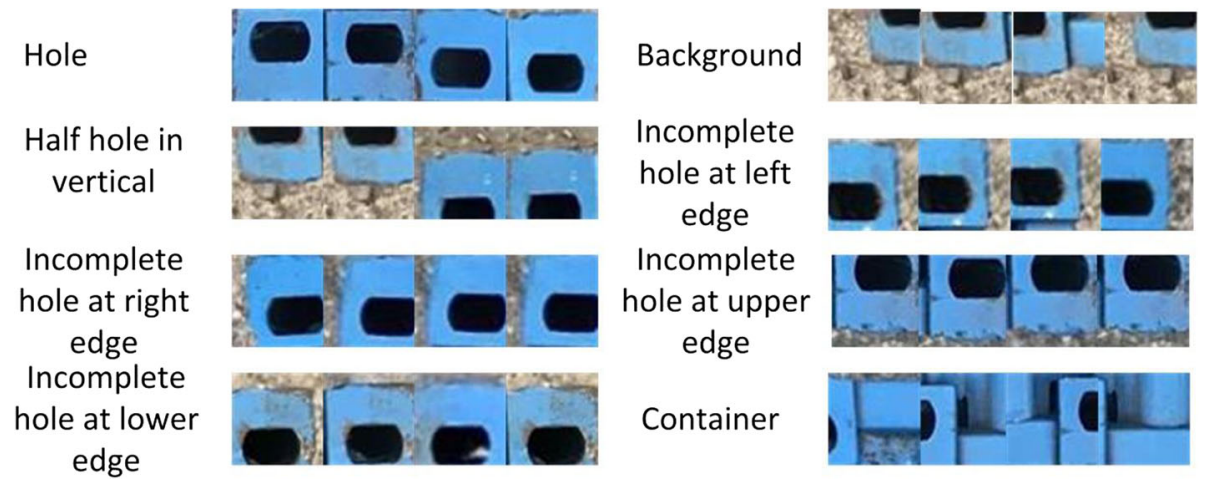

Fig. 3 Examples of data types

(1) At the corner point of the image, a $50 \times 50$ pixel sliding window was defined as the initial window.

(2) The HOG feature of the window was detected using the classification model, and if the prediction result was "hole," go to step (4); if the prediction result was "incomplete hole at upper/lower edge," stop sliding and go to step (3); if the prediction result was another classification result, slide the window $k_{1}$ pixels to the $X$-axis to predict again, until the window was outside the range of the block, then stop sliding and go to step (3).

(3) The sliding window first returned to the initial position of step (2). After sliding the window $k_{2}$ pixels to the $Y$-axis, the prediction was continued to step (2). If the prediction result was "hole," go to step (4). If the sliding window was outside the range of the block, stop sliding.

(4) Finally, the sliding windows, which were predicted as the "hole," were clipped from the top area image.

Based on this method, the holes figure can be clipped from the container top image.

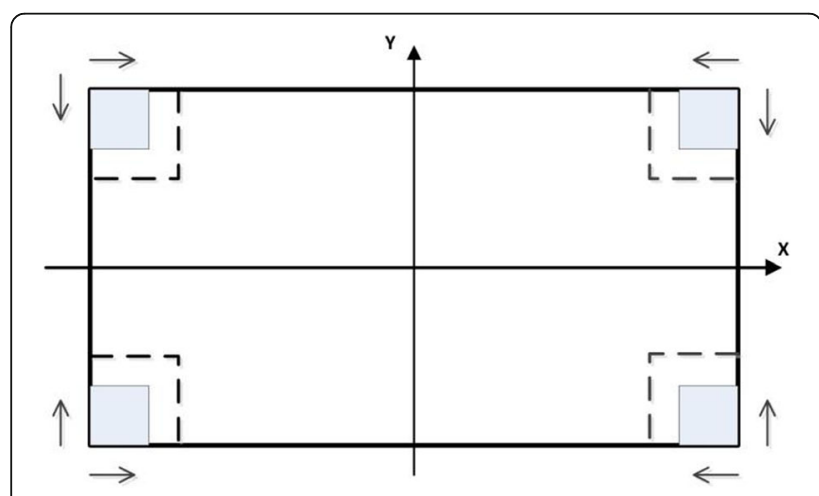

Fig. 4 Improved sliding window method

\subsection{The location of the lock holes}

To get the exact position of the lock holes, we need to detect the edge of the holes and find the contour center. After being converted to a grayscale image and getting the edges by using the Canny algorithm, the hole edge features were detected as follows. Firstly, the maximum area of all enclosing contours was found. If the maximum area was larger than $1 / 4$ of the image area, only the maximum contour is retained; otherwise, if the edge of the lock holes was not closed, the only unclosed contour with the largest perimeter was found and retained. In the end, the only remaining edge in the image was the contour of the lock holes.

Then, the least squares method was used to fit ellipses on the contour of the lock holes and the center of the holes on the sliding window were positioned. Finally, the center coordinates of four lock holes in the original image were calculated via Formula (6):

$$
\left\{\begin{array}{c}
\left(x_{h}^{(i)}, y_{h}^{(i)}\right)=\left(x_{h 2}^{(i)}, y_{h 2}^{(i)}\right)+\left(x_{s}^{(i)}, y_{s}^{(i)}\right)+\left(x_{r}, y_{r}\right), i=1,2,3,4 \\
\left(x_{h}^{\prime(i)}, y_{h}^{(i)}, 1\right)^{T}=R \times\left(x_{h}^{(i)}, y_{h}^{(i)}, 1\right)^{T}
\end{array}\right.
$$

Where $x_{h}^{(i)}, y_{h}^{(i)}$ is the coordinate of the center of the lock hole $i$ in the image after transformation. $x_{h 2}^{(i)}, y_{h 2}^{(i)}$ is the center of the lock hole coordinates within a clipped window. $x_{s}^{(i)}, y_{s}^{(i)}$ is the upper left corner coordinate of a clipped window within the container top area image. $\left(x_{n}\right.$ $y_{r}$ ) is the upper left corner coordinate of the container top area in the original image. $\mathrm{R}$ is the rotation matrix with the rotation angle $\theta$. Finally $x_{h}^{\prime(i)}, y_{h}^{\prime(i)}$ is the coordinate of holes center in the original image.

\section{Experiments}

\subsection{Preparation for experiments}

We validated the proposed method using two kinds of container miniatures, whose sizes were $1 / 15$ of the real $20 \mathrm{ft}$ container and $40 \mathrm{ft}$ container (In the following 
paper, the two container miniatures are named as " $20 \mathrm{ft}$ container" and " $40 \mathrm{ft}$ container."). Before testing our algorithm, it is necessary to create a training dataset that contains various conditions. The training dataset contains container feature extracted from various container images, which accounts for variability in position and the size of the container. Since the HOG features are less affected by illumination, the training dataset is collected on daytime. To reduce the interference of other features of the container to lock holes recognition, we made a strict distinction on the types of classification. The training dataset was divided into eight types, and the classification is detailed in Section 3.2.2.

After training, the testing dataset was randomly collected 1600 images. The testing dataset accounted for the variability in the form and size of the container, different position of a container placed, different lighting, and weather conditions. The setting of the testing dataset was as follows.

(1) The testing dataset was divided into four groups according to weather and lighting conditions, i.e., sunny, cloudy, rainy, and night. Each group was divided into two small groups according to the size of the container, i.e., $20 \mathrm{ft}$ container, $40 \mathrm{ft}$ container; each small group contains 200 images.

(2) The test used four $20 \mathrm{ft}$, eight $40 \mathrm{ft}$ container miniatures. In the lock holes area of the four $20 \mathrm{ft}$ container miniatures, one feature is completed, two are slightly rusted and the paint is fading, and one is severely rusted and the paint faded. In the lock holes area of eight $40 \mathrm{ft}$ container miniatures, two features are completed, four are slightly rusted and the paint faded, and two are severely rusted and the paint faded. To ensure the consistency across of each set of the testing dataset, we randomly assigned the number of each container miniature in a set of the testing dataset.

(3) The direction and location of the taken containers were random to simulate the error when the containers were placed in the station.

As can be seen in Table 1, there are four related references about the containers recognition and location: references [7, 8] mainly focused on container shape recognition and location, and $[9,10]$ mainly focused on lock holes recognition and location. Compared with the testing dataset from these references, some advantages of the proposed testing dataset were extracted. (1) The weather and lightness were more variable, and for the first time, the experiment was processed in rainy weather. (2) The test samples were diverse, including containers of different sizes and degrees of rust, and different placement angles. (3) We had the largest testing dataset, which reduced the error of the experiment results.

\subsection{Experiment results}

In experiments, block parameters $W_{1}$ and $W_{2}$ were all set to 75 and the step of sliding $k_{1}, k_{2}$ were all set to 4 . We assessed the detection performance by calculating the accuracy of 1600 samples. For recognition, it is a correct prediction if all the holes in an image are recognized correctly. The formula of accuracy is as follows:

$$
\text { accuracy }=\frac{\text { correct predictions }}{\text { allpredictions }}
$$

For locating, a correct prediction is defined by the following formula:

$$
\text { prediction }=\left\{\begin{array}{c}
1, \mid \text { error }_{v} \mid<1 \text { and } \mid \text { error }_{h} \mid<1 \text { pixel } \\
0, \mid \text { error }_{v} \mid>1 \text { or } \mid \text { error }_{h} \mid>1 \text { pixel }
\end{array}\right.
$$

Where error $v$ and error $_{h}$ are the vertical and horizontal error of the hole center between algorithm locating and manual locating respectively. It is a correct prediction if the prediction equals 1 . The formula of location accuracy is also defined via formula (7).

Table 2 shows the results of the experiments. In the daytime (sunny, cloudy), the average location accuracy is $98.13 \%, 95.25 \%$ in rainy days, and $90.50 \%$ at nights. The average recognition accuracy is $100 \%$. The $40 \mathrm{ft}$ container location accuracy is slightly lower than the $20 \mathrm{ft}$ container, because the rust and damage of the $40 \mathrm{ft}$ container keyhole surface are more serious than the $20 \mathrm{ft}$ container, which makes it difficult to locate the $40 \mathrm{ft}$ container lock holes.

The proposed method was compared with the two closely related references. Shen et al. [9] mainly focused on lock holes location, while Mi et al. [10] mainly focused on lock holes recognition. They all defined the

Table 1 Comparison with other datasets

\begin{tabular}{llllll}
\hline Reference & $\begin{array}{l}\text { Number of } \\
\text { testing dataset }\end{array}$ & $\begin{array}{l}\text { Number of weather } \\
\text { and lightness types }\end{array}$ & $\begin{array}{l}\text { Number of } \\
\text { container size }\end{array}$ & $\begin{array}{l}\text { Number of } \\
\text { container samples }\end{array}$ & Whether multi-angle \\
\hline Yoon et al. [8] & 1200 & 2 (sunny, cloudy) & 1 & 1 & No \\
Chen et al. [7] & 800 & 1 (daytime) & 1 & Unknown & Yes \\
Shen et al. [9] & 1000 & 2 (daytime, night) & 2 & Unknown & No \\
Mi et al. [10] & 500 & 1 (daytime) & 1 & Unknown & No \\
The proposed testing set & 1600 & 4 (sunny, cloudy, rainy, night) & 2 & 12 & Yes \\
\hline
\end{tabular}


Table 2 Experimental results

\begin{tabular}{llll}
\hline Weather/lightness & $\begin{array}{l}\text { Container } \\
\text { size (\%) }\end{array}$ & $\begin{array}{l}\text { Recognition } \\
\text { accuracy (\%) }\end{array}$ & $\begin{array}{l}\text { Location } \\
\text { accuracy (\%) }\end{array}$ \\
\hline Sunny & 20 & 100 & 99.5 \\
& 40 & 100 & 98.5 \\
Cloudy & 20 & 100 & 98.0 \\
& 40 & 100 & 96.5 \\
Rainy & 20 & 100 & 96.0 \\
& 40 & 100 & 94.5 \\
Night & 20 & 100 & 91.5 \\
& 40 & 100 & 89.5 \\
\hline
\end{tabular}

accuracy of the single lock hole (There were more than one hole in an image.). Thus, we used the same definition to record the experiment results. As shown in Table 3, the recognition and location accuracy in the daytime is close to $100 \%$, at night the location accuracy is $4.2 \%$ higher than Shen's. The improvement of accuracy is obvious.

\section{Discussion}

The study presented a comprehensive container dataset for container lock holes recognition and location. It also presents a new hybrid method that can automatically locate lock holes from an image of a complete container, which is taken from various viewpoints. In contrast with the other related studies' performance, the presented method shows the robustness to the rusty keyholes and changes of illumination as well as weather. Though the proposed method outperforms the state-of-the-art method, there is still room for improvement:

- Rust contour is connected with the edge of the lock holes during edge detection: when the lightness is bright (sunny, cloudy), most of the edge in images can be clearly detected, but the rust around the holes is easy to be connected with the contour of the hole, interfering with the shape of holes. To solve this problem, increasing the threshold of edge detection may be considered so that fewer pixels are preserved.

Table 3 Comparison with other results

\begin{tabular}{llll}
\hline Weather/lightness & Reference & $\begin{array}{l}\text { Lock holes } \\
\text { recognition } \\
\text { accuracy }\end{array}$ & $\begin{array}{l}\text { Lock holes } \\
\text { location } \\
\text { accuracy }\end{array}$ \\
\hline Daytime & Shen et al. [9] & $\backslash$ & $96.4 \%$ \\
& Mi et al. [10] & $97.6 \%$ & $\backslash$ \\
\multirow{2}{*}{ Night } & The proposed method & $100 \%$ & $99.53 \%$ \\
& Shen et al. [9] & $\backslash$ & $93.4 \%$ \\
& The proposed method & $100 \%$ & $97.6 \%$ \\
\hline
\end{tabular}

- Incomplete lock holes feature in edge detection: because of the weather conditions or rust around the holes, the hole contour may be broken or lose during edge detection. The only remaining contour can be incomplete hole edges, or even other features, such as corners. It is more likely to occur at night or on rainy days. In the rain, the detected edges are prone to breakage because of the accumulation of rainwater on the edge of the lock holes. At night, with the low illumination, the taken container features are not clear enough, hence to make the hole features easily miss. Besides, the brightness of the light during the night experiment is lower than the actual station. An enhancement in brightness or test in an actual environment will further improve the accuracy.

\section{Conclusions}

This paper presented a hybrid machine vision method for container lock holes recognition and location. The proposed approach is applicable to different placement angles and size of the container; it is also robust to rust around holes. The experiment results in recognition and location accuracy during the daytime are $100 \%$ and $98.13 \%$ respectively, which are higher than previous methods. In addition, the location accuracies in rainy days and night are $95.25 \%$ and $90.50 \%$ respectively, and the recognition accuracies are all $100 \%$, which proves that the method is feasible under special weather and light conditions. The solution of several research challenges, such as the detecting holes from multiple container surfaces, the variations of container angle, the low lighting conditions, and the special weather conditions, makes the method suitable for actual container terminals.

\section{Abbreviations}

HOG: Histograms of oriented gradients; SVM: Support vector machine; HSV: Hue, saturation, value; ECOC: Error-correcting output codes

\section{Acknowledgements \\ Thanks for the editors and reviewers. The authors gratelfully acknowledge} financial support from China Scholarship Council.

\section{Authors' contributions}

YD contributed to the algorithm, analyzed the data, and wrote the manuscript. $\mathrm{RD}$ contributed to the algorithm improvement and revised the manuscript. WC contributed to the experiment platforms. YW polished the manuscript. JZ contributed to the data collection. All authors read and approved the final manuscript.

\footnotetext{
Authors' information

Yunfeng Diao is currently working towards the Ph.D. degree in the Department of Engineer Machinery, Southwest Jiaotong University, China. His current research interest includes construction safety monitoring, human action recognition, and automatic container handling.

Wenming Cheng is a Professor and Head of Department of Engineer Machinery at Southwest Jiaotong University, China. His research interests include logistics equipment, industrial engineering, and intelligent optimization and simulation. He has published over 80 journals in China and abroad. He has received 3 national awards and more than 10 provincial awards in China. Run Du received the B.Sc. and Ph.D. degrees in mechanical engineering from Southwest Jiaotong University, Chengdu, China in 2003 and 2010. He is currently an assistant professor at Southwest Jiaotong
} 
University. His research interests include artificial intelligence in mechatronics and computational fluid dynamics. Prior to that, He had been a research engineer at ASM Pacific Technology Limited in Hong Kong SAR, China for 2 years.

\section{Funding}

This research is supported by the Sichuan Science and Technology Program under the Grand No.2019YFG0300, No.2019YFG0285, supported by Science and Technology Research and Development Program Key Issues for China Railway Chengdu and Wuhan Group Co., Ltd. nos. CX1804 and 18Y06.

\section{Availability of data and materials}

Please contact the corresponding author for data requests.

\section{Competing interests}

The authors declare that they have no competing interests.

\section{Author details}

'School of Mechanical Engineering, Southwest Jiaotong University, Chengdu, China. ${ }^{2}$ Technology and Equipment of Rail Transit Operation and Maintenance Key Laboratory of Sichuan Province, Chengdu, China. ${ }^{3}$ TStone Robotics Institute, The Chinese University of Hong Kong, Hong Kong SAR, China.

Received: 21 March 2019 Accepted: 16 June 2019

Published online: 26 June 2019

\section{References}

1. UTCAD, Review of Maritime Transport 2017. United Nation (2017)

2. J. He, Y. Huang, W. Yan, Yard crane scheduling in a container terminal for the trade-off between efficiency and energy consumption. Adv. Eng. Inform. 29, 59-75 (2015)

3. J.-t. Wang, T. Kong, J. Li, J.-g. Pan, Container alignment based on laser measurement and camera calibration. J. Syst. Simul. (China) 21, 4873-4878 (2009)

4. Q.H. Ngo, N.P. Nguyen, C.N. Nguyen, T.H. Tran, K.S. Hong, Fuzzy sliding mode control of container cranes. Int. J. Control. Autom. Syst. 13, 419-425 (2015)

5. Q.H. Ngo, K.S. Hong, Sliding-mode antisway control of an offshore container crane. IEEE-ASME. Trans. Mechatron. 17, 201-209 (2012)

6. L. Wei, L. Eung-Joo, Real-time container shape and range recognition for implementation of container auto-landing system. J. Korea. Multimed. Soc. 12, 794-803 (2009)

7. N. Chen, S. Wang, Z. Huang, Container recognition and location technology based on feature matching. J. Graph. 37, 530-536 (2016)

8. H.-J. Yoon, Y.-C. Hwang, E.-Y. Cha, leee, Real-time container position estimation method using stereo vision for container auto-landing system. International Conference on Control, Automation and Systems (Iccas 2010) (2010), pp. 872-876

9. Y. Shen, W. Mi, Z. Zhang, A positioning lockholes of container corner castings method based on image recognition. Polish. Marit. Res. 24, 95-101 (2017)

10. C. Mi, Z. Zhang, Y. Huang, Y. Shen, A fast automated vision system for container corner casting recognition. J. Mar. Sci. Technology-Taiwan. 24, 54-60 (2016)

11. N. Otsu, A threshold selection method from gray-level histograms. IEEE. Trans. Syst. Man. Cybern. SMC. 9, 62-66 (1979)

12. P. Soille, Morphological image analysis: principles and applications. SpringerVerlag (2003)

13. J. Macqueen, Some methods for classification and analysis of multivariate observations, Proc. of Berkeley Symposium on Mathematical Statistics and Probability1966, pp. 281-297

14. J. Canny, A computational approach to edge detection. IEEE. Trans. Pattern. Anal. Mach. Intell. 8, 679-698 (1986)

15. N. Dalal, B. Triggs, in 2005 IEEE Computer Society Conference on Computer Vision and Pattern Recognition, Vol 1, Proceedings, leee Computer Soc, Los Alamitos, ed. by C. Schmid, S. Soatto, C. Tomasi. Histograms of oriented gradients for human detection (2005), pp. 886-893

16. C.W. Hsu, C.J. Lin, A comparison of methods for multiclass support vector machines. leee. Trans. Neural. Netw. 13, 415-425 (2002)

17. S. Escalera, O. Pujol, P. Radeva, On the decoding process in ternary error-correcting output codes. IEEE. Trans. Pattern. Anal. Mach. Intell. 32, 120-134 (2010)

\section{Publisher's Note}

Springer Nature remains neutral with regard to jurisdictional claims in published maps and institutional affiliations.

\section{Submit your manuscript to a SpringerOpen ${ }^{\circ}$ journal and benefit from:}

- Convenient online submission

- Rigorous peer review

- Open access: articles freely available online

High visibility within the field

- Retaining the copyright to your article

Submit your next manuscript at $\boldsymbol{\nabla}$ springeropen.com 